\title{
Benchmark and Mean-Variance Problems for Insurers
}

\author{
Nicole Bäuerle \\ Institute for Mathematical Stochastics \\ University of Hannover, Germany \\ D-30167 Hannover, Germany \\ e-mail: baeuerle@stochastik.uni-hannover.de
}

\begin{abstract}
We consider the classical Cramér-Lundberg model with dynamic proportional reinsurance and solve the problem of finding the optimal reinsurance strategy which minimizes the expected quadratic distance of the risk reserve to a given benchmark. This result is extended to a mean-variance problem.
\end{abstract}

Key words: stochastic LQ problem, Lagrange theory, HJB equation

\section{Introduction}

A milestone in mathematical finance is Markowitz's quantitative approach to the problem of wealth allocation among a number of (risky) assets in order to achieve a given expected return with minimal variance. This problem has also become well-known under the name mean-variance portfolio problem. The contribution of the present paper is to point out that this criterion could also be of interest in insurance applications. For example a static mean-variance approach can be used to obtain the optimal form of reinsurance (see e.g. Kaluszka (2004)). In the present paper we investigate a simple (dynamic) Cramér-Lundberg model with proportional reinsurance and solve the problem of finding the optimal reinsurance strategy which minimizes the expected quadratic distance of the risk reserve to a given benchmark. This result can then be used to solve the corresponding mean-variance problem, i.e. to find the optimal reinsurance strategy which minimizes the variance of the risk reserve under the condition that the expected risk reserve reaches a certain benchmark. This question is also part of the dynamic financial analysis of an insurance company.

By now there exist numerous papers on the mean-variance problem and its extensions in finance. Among others, Zhou/Li (2000) (see Zhou (2003) for an overview) investigate the continuous-time version of this problem where stock prices follow a diffusion process. Mean-variance problems with jump-diffusions have been investigated in Øksendal/Sulem (2005), chapter 3.3 (see also Framstadt et al. (1999)) and Guo/Xu (2004). So far all the applications focus on classical financial portfolio allocation problems. In the simple reinsurance model we consider, we assume non-negativity constraints on the control. Because of this additional feature we were not able to use the previously published results. In Li et al. (2002) the authors also deal with non-negativity constraints on the control but their model is a pure diffusion. Essentially the benchmark problem we consider is a linear-quadratic ( $L Q)$ problem and there are at least three different solution techniques: (i) completion of squares (see e.g. Zhou/Li (2000)), (ii) the Hamiltonian approach (see e.g. Øksendal/Sulem (2005)), (iii) the approach via the Hamilton-Jacobi-Bellman (HJB) equation (see e.g. Guo/Xu (2004)). Due to the non-negativity constraints on the control we solve our problem with the last approach and the Langrange theory.

The paper is organized as follows. In Section 2 the reinsurance model is introduced and a benchmark optimization problem is formulated. Section 3 contains the solution of the benchmark problem. This result is then extended in section 4 to the mean-variance problem. 


\section{A Benchmark Problem for the Risk Reserve Process}

We consider the random evolution of the risk reserve of an insurance company like in the Cramér Lundberg model. This means that claims arrive according to a Poisson process $\left(N_{t}\right)$ with intensity $\lambda>0$ and that the claim sizes $Z_{1}, Z_{2}, \ldots$ are independent and identically distributed with distribution $Q$. We assume that $\mu:=\int z Q(d z)$ and $\mu_{2}:=\int z^{2} Q(d z)$ are both finite. Premiums are calculated according to the expectation principle and the premium rate is thus given by $\left(1+\eta_{1}\right) \lambda \mu$, where $\eta_{1}>0$ is the safety load of the insurance company. The risk reserve at time $t \geq 0$ is then defined by

$$
X_{t}:=x_{0}+\left(1+\eta_{1}\right) \lambda \mu t-\sum_{n=1}^{N_{t}} Z_{n}
$$

where $x_{0}>0$ is the initial risk reserve.

Throughout, we assume that $\left(\Omega, \mathcal{F}, \mathfrak{F}=\left\{\mathcal{F}_{t}, 0 \leq t \leq T\right\}, P\right)$ is a filtered probability space, all appearing processes are adapted w.r.t. $\mathfrak{F}$ and $T>0$ is a fixed time horizon.

The risk reserve process can now be controlled by the insurance company by effecting a proportional reinsurance and/or by acquiring new business. We define by

$$
U:=\left\{u=\left(u_{s}\right)_{0 \leq s \leq T} \mid u_{s} \geq 0,\left(u_{s}\right) \text { is } \mathfrak{F} \text { - predictable, }\left(u_{s}\right) \text { is bounded from above }\right\}
$$

the set of all admissible reinsurance strategies over the time horizon $[0, T]$. The premium rate for the reinsurance product is given by $\left(1+\eta_{2}\right) \lambda \mu\left(1-u_{t}\right)$ where $\eta_{2}>0$ is the safety load of the reinsurer. Thus, the risk reserve under control $u \in U$ is given by

$$
X_{t}^{u}=x_{0}+\left(1+\eta_{1}\right) \lambda \mu t-\left(1+\eta_{2}\right) \lambda \mu \int_{0}^{t}\left(1-u_{s}\right) d s-\sum_{n=1}^{N_{t}} Z_{n} u_{T_{n}}
$$

where $T_{1}, T_{2}, \ldots$ are the claim arrival time points. If we introduce $\theta:=\left(\eta_{2}-\eta_{1}\right) \lambda \mu$ and $c:=$ $\left(1+\eta_{2}\right) \lambda \mu$, then

$$
X_{t}^{u}=x_{0}-\theta t+c \int_{0}^{t} u_{s} d s-\sum_{n=1}^{N_{t}} Z_{n} u_{T_{n}} .
$$

Note that $u_{s} \in[0,1]$ corresponds to a reinsurance cover and $u_{s}>1$ corresponds to acquiring new business. Typically $\theta>0$, but we do not need to assume this here. The aim now is to control the risk reserve in such a way that it is at time $T$ close to a certain predefined benchmark $b \in \mathbb{R}_{+}$. When we measure deviations from $b$ by the squared distance we arrive at the following constraint optimization problem:

$$
(B M)\left\{\begin{array}{c}
E^{x_{0}}\left(X_{T}^{u}-b\right)^{2} \\
u \in U
\end{array} \rightarrow \min \right.
$$

where $E^{x_{0}}$ is the expectation given that we start at time 0 with risk reserve $x_{0} \in \mathbb{R}$. This is a so-called LQ-problem. Problems of this type have been studied extensively already. However we were not able to use a general result like e.g. in Øksendal/Sulem (2005) chapter 3.3 since we have non-negativity constraints on the control variable. In Li et al. (2002) the authors also deal with non-negativity constraints on the control but their model is a pure diffusion. In the next section we show how to solve this problem with the help of the HJB-equation. To this end, we define by

$$
V(t, x):=\inf _{u \in U} E\left[\left(X_{T}^{u}-b\right)^{2} \mid X_{t}^{u}=x\right]
$$

the value function of the problem for $t \in[0, T]$ and $x \in \mathbb{R}$. Obviously we have $V(T, x)=(x-b)^{2}$. 


\section{The HJB Equation and a Verification Theorem}

The HJB equation of this problem is

$$
0=\min _{u \geq 0}\left\{v_{t}+v_{x}(c u-\theta)+\lambda \int v(t, x-u z)-v(t, x) Q(d z)\right\}
$$

with boundary condition $v(T, x)=(x-b)^{2}$. We will first show that there exists a classical smooth solution of the HJB equation which is in contrast to the results in Li et al. (2002). There the authors had to deal with viscosity solutions to solve the problem. At the end of this section we will verify that the solution of (1) is the solution of our control problem. In what follows we define the two regions

$$
\begin{aligned}
& U_{b}:=\left\{(t, x) \in[0, T] \times \mathbb{R} \mid x \geq-A_{b}(t)\right\} \\
& D_{b}:=\left\{(t, x) \in[0, T] \times \mathbb{R} \mid x<-A_{b}(t)\right\}
\end{aligned}
$$

where $A_{b}(t):=\theta(t-T)-b$.

Theorem 1: (Solution of the HJB equation) The following function $v \in C^{1,1}$ is a solution of the HJB equation (1):

$$
v(t, x)=\left\{\begin{aligned}
\left(x+A_{b}(t)\right)^{2}, & \text { for }(t, x) \in U_{b} \\
e^{\rho(t-T)}\left(x+A_{b}(t)\right)^{2}, & \text { for }(t, x) \in D_{b}
\end{aligned}\right.
$$

where $\rho=\frac{(c-\lambda \mu)^{2}}{\lambda \mu_{2}}$ and $A_{b}(t)=\theta(t-T)-b$. The minimum points $u^{*}(t, x)$ are given by

$$
u^{*}(t, x)=\left\{\begin{aligned}
0, & \text { for }(t, x) \in U_{b} \\
\frac{\lambda \mu-c}{\lambda \mu_{2}}\left(x+A_{b}(t)\right), & \text { for }(t, x) \in D_{b}
\end{aligned}\right.
$$

Proof: It is easy to see that $v \in C^{1,1}$. Note in particular that on $\left\{(t, x) \in[0, T] \times \mathbb{R} \mid x=-A_{b}(t)\right\}$ we have $v(t, x)=v_{t}(t, x)=v_{x}(t, x)=0$. Obviously the value function satisfies the boundary condition and is for all $(t, x)$ of the form

$$
v(t, x)=P(t) x^{2}+Q(t) x+R(t)
$$

Plugging this into the HJB equation gives

$$
0=\min _{u \geq 0}\left\{P_{t} x^{2}+Q_{t} x+R_{t}+(2 P x+Q)((c-\lambda \mu) u-\theta)+P \lambda \mu_{2} u^{2}\right\} .
$$

In order to show that $v$ solves this problem we have to use the Karush-Kuhn-Tucker conditions. The Lagrange function of this problem is

$$
L(u, y)=u^{2} P \lambda \mu_{2}+(2 P x+Q)(c-\lambda \mu) u-y u,
$$

where $y \geq 0$ is the Lagrange multiplier. $u^{*}$ is the minimizing point in (2) if and only if there exists an $y^{*}$ such that the following Karush-Kuhn-Tucker conditions are satisfied:

1. $\frac{\partial L}{\partial u}=0 \Leftrightarrow 2 u^{*} P \lambda \mu_{2}+(2 P x+Q)(c-\lambda \mu)-y^{*}=0$.

2. $y^{*} u^{*}=0$

3. $y^{*}, u^{*} \geq 0$. 
Case 1: $(t, x) \in U_{b}$

In this case we have to choose $u^{*}=0$ and $y^{*}=(2 P x+Q)(c-\lambda \mu)$ which yields 1 . and 2 . Inserting the expressions for $P, Q$ and $R$ gives that $y^{*} \geq 0$ if and only if $x \geq-A_{b}(t)$ which is satisfied since $(t, x) \in U_{b}$.

Case 2: $(t, x) \in D_{b}$

In this case we have to choose $y^{*}=0$ and $u^{*}=\frac{(2 P x+Q)(\lambda \mu-c)}{2 P \lambda \mu_{2}}$ which yields 1 . and 2. Inserting the expressions for $P, Q$ and $R$ we obtain $u^{*}=\frac{\lambda \mu-c}{\lambda \mu_{2}}\left(x+A_{b}(t)\right)$. Since $c>\lambda \mu$ and $(t, x) \in D_{b}$ we have $u^{*}>0$.

The validity of the HJB equation in both cases follows directly by inserting the expressions for $P, Q, R$ and $u^{*}$.

Finally we verify that the solution of the HJB equation given in Theorem 1 is indeed the solution of our control problem.

Theorem 2: (Verification Theorem) Let $v$ and $u^{*}$ be given as in Theorem 1 . It holds that $V(t, x)=v(t, x)$ for all $(t, x) \in[0, T] \times \mathbb{R}$ and the optimal control $u^{*}=\left(u_{t}^{*}\right)$ is given in feedback form by $u_{t}^{*}=u^{*}\left(t, X_{t}^{*}\right)$ where $\left(X_{t}^{*}\right)$ is the risk reserve under $u^{*}$.

Proof: Let $u \in U$ be arbitrary. Applying Itô's Lemma to $v \in C^{1,1}$ and $\left(X_{t}^{u}\right)$ we obtain:

$$
\begin{aligned}
v\left(T, X_{T}^{u}\right)= & v(t, x)+\int_{t}^{T} v_{t}\left(s, X_{s}^{u}\right)+v_{x}\left(s, X_{s}^{u}\right)\left(c u_{s}-\theta\right) d s+ \\
& \int_{t}^{T} \int_{\mathbb{R}_{+}}\left(v\left(s, X_{s-}^{u}-u_{s} z\right)-v\left(s-, X_{s-}^{u}\right)\right) M(d s, d z)+ \\
& +\lambda \int_{t}^{T} \int_{\mathbb{R}_{+}}\left(v\left(s, X_{s-}^{u}-u_{s} z\right)-v\left(s-, X_{s-}^{u}\right)\right) Q(d z) d s
\end{aligned}
$$

where $N([0, t] \times B):=\sum_{n \in \mathbb{N}} I_{\left[T_{n} \leq t, Z_{n} \in B\right]}$ is the Poisson random measure and $M(d t, d z)=$ $N(d t, d z)-\lambda d t Q(d z)$ a martingale w.r.t. $\mathfrak{F}$. Making use of the fact that $v$ satisfies the HJB equation gives

$$
v\left(T, X_{T}^{u}\right) \geq v(t, x)+\int_{t}^{T} \int_{\mathbb{R}_{+}}\left(v\left(s, X_{s-}^{u}-u_{s} z\right)-v\left(s-, X_{s-}^{u}\right)\right) M(d s, d z)
$$

and equality is obtained in the case $u=u^{*}$. Note that

$$
E^{x_{0}}\left[\int_{0}^{t} \int_{\mathbb{R}_{+}}\left|v\left(s, X_{s-}^{u}-u_{s} z\right)\right| Q(d z) d s\right]<\infty
$$

and thus the integral process is a martingale with zero expectation. Since $v(T, x)=(x-b)^{2}$, taking the conditional expectation on both sides yields

$$
\begin{aligned}
& E\left[\left(X_{T}^{u}-b\right)^{2} \mid X_{t}=x\right] \geq v(t, x) \\
& E\left[\left(X_{T}^{*}-b\right)^{2} \mid X_{t}=x\right]=v(t, x)
\end{aligned}
$$

which implies the statement.

\section{A Mean-Variance Problem with Constraints}

In this section we consider as in the classical Markowitz setting the aim of the insurance company to minimize the risk of the terminal reserve measured by the variance over all admissible 
reinsurance strategies which yield the same expected terminal reserve $b \in \mathbb{R}$. This problem can be formulated as

$$
(M V)\left\{\begin{array}{c}
E^{x_{0}}\left(X_{T}^{u}-b\right)^{2} \rightarrow \min \\
E^{x_{0}} X_{T}^{u}=b \\
u \in U
\end{array}\right.
$$

The value of this problem is denoted by $J\left(x_{0}\right)$. This problem can be solved via the well-known Lagrange multiplier technique. To this end, we define for $y \in \mathbb{R}$

$$
\begin{aligned}
J_{u}\left(x_{0}, y\right) & :=E^{x_{0}}\left[\left(X_{T}^{u}-b\right)^{2}+2 y\left(X_{T}^{u}-b\right)\right] \\
& =E^{x_{0}}\left[\left(X_{T}^{u}-(b-y)\right)^{2}\right]-y^{2}
\end{aligned}
$$

The duality theory implies

$$
J\left(x_{0}\right)=\sup _{y \in \mathbb{R}} \inf _{u \in U} J_{u}\left(x_{0}, y\right) .
$$

$\inf _{u \in U} J_{u}\left(x_{0}, y\right)$ has been solved in section 2. Thus, it remains to find $y^{*} \in \mathbb{R}$ which maximizes $y \mapsto \inf _{u \in U} J_{u}\left(x_{0}, y\right)$. The insurance strategy which yields the solution of problem $(M V)$ is called efficient for return $b .\left(b, \sqrt{V\left(x_{0}\right)}\right) \in \mathbb{R}^{2}$ is called efficient point and the set of all those points is called efficient frontier. Thus, the efficient frontier gives the line of the minimum risk reserve standard deviation as a function of the expected return. It is usually nicely illustrated in text books on the classical Markowitz problem (see e.g. Luenberger (1998)). We obtain the following statement:

Theorem 3: Assume that $x_{0} \leq b+\theta T$. Then the optimal reinsurance strategy for the meanvariance problem is given by $u_{t}^{*}=u^{*}\left(t, X_{t}^{*}\right)$ where

$$
u^{*}(t, x)=\frac{c-\lambda \mu}{\lambda \mu_{2}}\left(\frac{x_{0}-e^{\rho T}(b+\theta T)}{1-e^{\rho T}}-\theta t-x\right) .
$$

The efficient frontier is given by

$$
\operatorname{Var} X_{T}^{*}=\left(x_{0}-\theta T-b\right)^{2} \frac{1}{e^{\rho T}-1} .
$$

Proof: The solution of $\inf _{u \in U} J_{u}\left(x_{0}, y\right)$ is given in Theorem 1 where we have to replace $b$ by $b-y$. Suppose first that $x_{0} \leq \theta T+b-y$. Note that in this case the region $D_{b-y}$ is never left, since whenever the line $-A_{b-y}(t)$ is reached, we set $u^{*}=0$ (full reinsurance) for the remaining period and arrive at time $T$ in $b-y$. Thus we have

$$
\inf _{u \in U} J_{u}\left(x_{0}, y\right)=e^{-\rho T}(x-\theta T-b+y)^{2}-y^{2} .
$$

Minimizing this expression over $y$ (note that it is concave) gives $y^{*}=\frac{b+\theta T-x_{0}}{1-e^{\rho T}}$. Inserting $y^{*}$ in the result of Theorem 1 gives the solution of the mean-variance problem:

$$
\begin{gathered}
u^{*}(t, x)=-\frac{c-\lambda \mu}{\lambda \mu_{2}}\left(x+A_{b-y^{*}}(t)\right) \\
\operatorname{Var} X_{T}^{*}=e^{-\rho T}\left(x_{0}+A_{b-y^{*}}(0)\right)^{2}-\left(y^{*}\right)^{2} .
\end{gathered}
$$

Note also that $x_{0} \leq \theta T+b-y^{*}$ is equivalent to $x_{0} \leq \theta T+b$.

Though the solution of the quadratic benchmark problem in Theorem 1 consists of two regions, the solution of the mean-variance problem does not. The reason is the assumption $x_{0} \leq b+\theta T$ which ensures that the constraint $E^{x_{0}} X_{T}^{u}=b$ can be satisfied. Under this assumption the down 
region $D_{b-y^{*}}$ is never left by the risk reserve process, since once the boundary $x=-A_{b}(t)$ is reached, we apply full reinsurance $\left(u^{*}=0\right)$ for the remaining time and the risk reserve falls on the straight line to $b$ at time $T$. Note also that the efficient frontier is a straight line as in the case of mean-variance portfolio selection with a risk free asset.

In conclusion, the model is rather simple, however, in the LQ-setting the problem can easily be solved and the solution is quite simple and depends only on the first and second moment of the claim sizes. Moreover, it does not matter if one wants to solve a diffusion or a jump(-diffusion) problem. Both are equally easy to solve which is not the case for other risk measures. As long as one stays in the LQ-setting even much more general models can be solved.

Acknowledgement: I am grateful to my $\mathrm{PhD}$ advisor Ulrich Rieder for introducing me into the theory of stochastic control and mathematical finance. I dedicate this paper to him on the occasion of his 60ies birthday.

\section{References}

[1] N. C. Framstad, B. Øksendal and A. Sulem (1999), "Optimal consumption and portfolio in a jump diffusion market", in: Shiryaev, A. et al. (eds.) Workshop on mathematical finance. Paris: INRIA, pp. 9-20.

[2] W. Guo and C. Xu (2004), "Optimal portfolio selection when stock prices follow an jumpdiffusion process", Mathem. Methods of Oper. Res. 60, 485 - 496.

[3] M. Kaluszka (2004), "An extension of Arrow's result on optimality of a stop-loss contract", Ins. Mathem. \& Econ. 35, 527 - 536.

[4] X. Li, X. Zhou and A. Lim (2002), "Dynamic mean-variance portfolio selection with noshorting constraints", SIAM J. Control Optim. 40, 1540-1555.

[5] D.G. Luenberger (1998), Investment Science. Oxford University Press, New York.

[6] B. Øksendal and A. Sulem (2005), Applied stochastic control of jump diffusions. SpringerVerlag, Berlin.

[7] X.Y. Zhou (2003), "Markowitz's world in continuous time and beyond", in: Yao, D.D. et al. (eds.) Stochastic modeling and optimization, pp. 279-309. Springer-Verlag, New York.

[8] X.Y. Zhou and D. Li (2000), "Continuous-time mean-variance portfolio selection: a stochastic LQ framework", Appl. Math. Optim. 42, pp. 19-33. 